\title{
Effects of fiber treatment on morphology, tensile and thermogravimetric analysis of oil palm empty fruit bunches fibers
}

\begin{abstract}
The objective of this study was to evaluate the effect of fiber treatment on both morphological and single fiber tensile strength of empty fruit bunch (EFB). EFB fiber was treated with boiling water, $2 \%$ sodium hydroxide $(\mathrm{NaOH})$ and combination both $\mathrm{NaOH}$ and boiling water. Fiber morphology was characterized by scanning electron microscopy (SEM). Thermogravimetric analysis (TGA) was further used to measure the amount and rate of change in the weight (weight loss) of treated fiber as a function of temperature. Based on the results of this work it seems that alkali treatment improved most of the fiber properties. $\mathrm{NaOH}$ treatment was found to alter the characteristic of the fiber surface topography as seen by the SEM. The thermal stability of $\mathrm{NaOH}$ treated and water boiling treated EFB fiber was found to be significantly higher than untreated fiber. The best results were obtained for alkali treated fiber where the tensile strength and Youngôs modulus increased compared to untreated fibers. The overall results showed that alkali treatment on EFB fiber enhanced the tensile strength and thermal stability of the fiber samples.
\end{abstract}

Keyword: A. Fibers; D. Electron microscopy; B. Thermal properties; D. Thermal analysis 